\title{
Management of cytomegalovirus antibody negative patients undergoing heart transplantation
}

\author{
R Freeman, F K Gould, A McMaster
}

\begin{abstract}
In a series of 61 consecutive patients undergoing heart, heart and lung, and lung transplantation, 24 patients were known to be cytomegalovirus (CMV) antibody negative on the day of transplantation. Enzyme linked immunosorbent assays (ELISA) for CMV IgG were performed on donor samples on the day of operation. In 16 of the 24 susceptible patients the test was negative and the only preventive measure taken was the use of blood and blood products from CMVantibody negative blood donors. None of these patients acquired primary infection with CMV. In another six patients the donor serum was found to contain CMV specific IgG, and in these patients, including one heart and lung transplant recipient, prophylaxis with $\mathrm{CMV}$ specific hyperimmune globulin was given. All six patients developed CMV IgM antibodies and in five there was an associated but clinically mild illness. None of these patients required treatment. In the remaining two patients ELISA tests on the donor sera gave equivocal results and hyperimmune globulin was witheld. Both patients developed primary CMV infection of greater severity than those given hyperimmune globulin and one required treatment. Reference tests confirmed that the donor sera contained CMV antibodies.

Primary CMV infection in susceptible patients after heart transplantation can be avoided by the use of screened blood and blood products where the organ donor is seronegative to $\mathrm{CMV}$ and it can be improved by the use of prophylactic hyperimmune globulin where the donor is CMV antibody positive.
\end{abstract}

Cytomegalovirus (CMV) is a well recognised cause oî morbidity and mortality after solid organ transplantation. Primary infection with $\mathrm{CMV}$ is associated with the more serious forms of the disease, such as pneumonitis, oesophagitis, colitis and retinitis, and is more likely to be fatal than CMV infection due to reactivation.

In primary infection the virus can be introduced into a susceptible - that is, CMV antibody negative-recipient by blood and blood products from CMV antibody positive blood donors, ${ }^{2}$ or it can be transmitted by the graft if the organ donor is CMV antibody positive. ${ }^{1}$
Early symptoms of primary CMV may be nonspecific, and although advances have been made in the rapid diagnosis of the infection, ${ }^{3}$ the patient may be severely ill before specific drugs are given. Treatment is not always effective and may itself produce serious side effects. ${ }^{4}$ The occasions when CMV will be introduced are predictable and identifiable, and a strategy aimed at prevention of CMV transmission is therefore a logical approach to the management of CMV antibody negative recipients.

Transmission by blood or blood products can theoretically be eliminated by ensuring that only CMV antibody negative blood and organ transplants are used in susceptible patients: ${ }^{2} \mathrm{~A}$ similar approach to CMV transmitted by graft is less attractive as donor supplies are insufficient to match always a CMV antibody negative donor to a susceptible recipient. This is especially true in heart transplantation where there is no long term supportive treatment available to maintain the patient until a suitable donor is found. An alternative approach is to implant the CMV positive graft while starting prophylaxis with CMV specific hyperimmune globulin. ${ }^{5}$ We combined both these approaches (screening of blood donations and use of hyperimmune globulin) in the management of CMV antibody negative patients undergoing cardiac transplantation.

\section{Methods}

Between May 1985 and June 1988 a total of 61 patients underwent heart, heart and lung, or single lung transplantation in this unit. The CMV specific IgG antibody state of all the patients had been determined at the time of acceptance for transplantation by enzyme linked immunosorbent assay (ELISA), and 24 patients ( 23 cardiac transplantation candidates and one heart and lung transplantation candidate) were deemed susceptible to CMV on the day of operation.

Serum from each donor was obtained at the time of organ retrieval and tested in parallel on the day of operation with a fresh sample from the recipient for the presence of CMV specific IgG. Depending on the results of these tests, the following strategy was followed:

Group $A$ donor and recipient CMV negative: In these patients all blood and blood products used at operation and in the postoperative period were known to be CMV antibody negative. No other measures to prevent CMV infection were used. 
Group $B$ donor CMV positive and patient $C M V$ negative: In addition to the use of $\mathrm{CMV}$ antibody negative blood and blood products, each patient was given prophylaxis with CMV specific hyperimmune globulin, $100 \mathrm{mg} / \mathrm{kg}$ intravenously, on days $0,7,14,21,28$ and day 49 after surgery.

All patients were observed clinically for any sign of CMV infection over a period of at least three months. In the absence of any evidence of infection a serum sample was taken at weekly intervals for the first month and monthly intervals for the first three months. Tests for CMV specific IgG were carried out after three months. A positive test at this stage prompted testing for CMV specific IgM antibody on the earlier samples. Clinical symptoms suspicious of $\mathrm{CMV}$ infection prompted the taking of serum samples at weekly intervals which were tested for CMV specific IgM antibody. Clinical disease associated with the appearance of CMV specific IgM was attributed to primary infection with CMV and the severity was assessed by a previously described scoring system. $^{6}$

\section{IgG AND IgM TESTS}

CMV IgG was detected using a commercial kit supplied by Northumbria Biologicals Ltd (Virenz G CMV, Northumbria Biologicals, Cramlington, Northumbria) and CMV specific IgM was detected using Vironostika IgM kits (Organon Ltd, Cambridge). In both instances procedures were carried out according to the manufacturer's instructions. Borderline results were repeated on at least one further testing occasion and if doubt persisted the specimen was sent for testing by a non-ELISA technique at a reference laboratory.

\section{TESTING OF BLOOD DONATIONS}

This was carried out at the Northern Regional Blood Transfusion Centre. Donors were tested for CMV antibody at the time of donation by a latex agglutination method (CMV Scan; Beckton-Dickinson UK Ltd), performed according to the manufacturer's instructions.

\section{SPECIFIC HYPERIMMUNE GLOBULIN}

With one exception, each patient's course was supplied by the National Blood Transfusion Service using products processed at the Scottish Protein Fractionation Centre, Edinburgh. The immunoglobulin is made from the plasma from donors with an antibody titre of greater than 1 in 32 by direct immunofluorescence testing and the final product has 10 times the anti-CMV potency of normal immunoglobulin when tested by an ELISA technique. In one instance a commercial alternative (Cytotect; Biotest-Pharmia Ltd, Dreitch, West Germany) was used. Neither preparation contains CMV specific IgM antibodies.

\section{Results}

Of the 16 patients who received a CMV negative graft and who were given CMV negative blood products, four died within two weeks of operation. None of the 12 survivors developed any illness suggestive of CMV infection or developed CMV specific IgG or IgM during the ensuing three months. Eight patients (seven with cardiac transplants and one with a heart and lung transplant) received grafts from CMV positive donors (table). In cases 2 and 4 the ELISA test gave borderline results on repeated testing and prophylactic imrhune globulin was withheld. Reference laboratory results eventually confirmed the positivity of the donors and these patients can be regarded as having unmodified disease. Case 2 developed fever, muscle pains, and abdominal pain. Renal function was impaired, although never to the extent of requiring dialysis, and concurrent cyclosporin A treatment was able to be continued. The patient eventually recovered without any specific treatment, although the illness attributed to cytomegalovirus infection lasted for 13 days before resolving.

Persisting fever and malaise in case 4 with evidence of liver disturbance and deterioration in renal function led to treatment with disodium phosphonoformate (Foscarnet). This drug was not well tolerated and was withdrawn after seven days. Intravenous CMV specific hyperimmune globulin $(100 \mathrm{mg} / \mathrm{kg})$ was given on two successive days and again one week later. The patient then went on to make a full recovery. Again it was possible to maintain cyclosporin treatment throughout the illness. In both of these patients the diagnosis was confirmed by the appearance of CMV specific IgM antibody during the later stages of the clinical illness or in convalescence.

In the remaining six patients a full course of prophylactic immunoglobulin was given. The severity of disease and the postoperative interval to the first detection of CMV specific IgM antibody is given in the table. Two cases

Onset relative to transplantation, clinical severity, and interval to appearance of serum CMV specific IgM antibody in eight susceptible patients receiving grafts from CMV antibody positive donors

\begin{tabular}{llll}
\hline Case No & $\begin{array}{l}\text { Onset of } \\
\text { illness (days) }\end{array}$ & Clinical score & $\begin{array}{l}\text { Detection of IgM (days } \\
\text { after transplantation) }\end{array}$ \\
\hline 1 & 45 & 3 & 48 \\
$2^{\star}$ & 60 & 8 & 66 \\
3 & 32 & 4 & 45 \\
$4^{\star}$ & 21 & 7 & 62 \\
5 & 42 & 0 & 53 \\
$6 \dagger$ & 30 & 4 & 42 \\
7 & 29 & 2 & 59 \\
8 & $32 \cdot 4$ & 3 & 67 \\
Mean (all) & $29 \cdot 6$ & $2 \cdot 8$ & $55 \cdot 2$ \\
Mean (globulin given) & $2 \cdot 6$ & $52 \cdot 2$ \\
\hline
\end{tabular}

*Both these patients were not given prophylactic globulin

†This patient underwent heart and lung transplantation. 
merit brief comment. Case 6 was a heart and lung transplant recipient who had been discharged from hospital without having developed any symptoms suggestive of CMV infection. Before her discharge she had undergone bronchoalveolar lavage (BAL) and transbronchial lung biopsy (TBLB) for the diagnosis of rejection and CMV was not present in these early specimens. On the forty second day after transplantation she was noted to have radiological pneumonitis and her pulmonary function had deteriorated. None the less she continued to have a normal exercise tolerance. BAL and TBLB were performed and $C M V$ was isolated from all samples. CMV pneumonitis was present in the biopsy specimen. No specific treatment was given and she received the final (day 49) dose of prophylactic immunoglobulin and made an uncomplicated recovery as an outpatient, including the rapid resolution of the radiological changes. CMV specific IgM was present in blood samples taken on the day of the biopsy which showed histological evidence of CMV pneumonitis. Case 5 was a cardiac transplant recipient who received a CMV positive graft. Despite the development of CMV specific IgM on day 53 she failed to exhibit any signs or symptoms which could be attributed to CMV infection.

\section{Discussion}

Our study shows that the incidence and severity of primary CMV infection after heart transplantation can be influenced by appropriate measures. The routine use of CMV antibody negative blood products in susceptible patients receiving a graft from a CMV antibody negative donor can prevent primary CMV infection. In one recent study in which unscreened blood products were used in an identical setting primary CMV infection occurred in $27 \%$ of the patients. ${ }^{7}$ Our experience strongly supports the conclusion of those authors that seronegative blood products should be routinely used in such patients. Our results also agree with a recent report that blood products screened for CMV prevent transmission by this route after bone marrow transplantation. ${ }^{2}$

Secondly, our limited experience with prophylactic CMV specific hyperimmune globulin suggests that the primary CMV infection which results from the implantation of a positive graft into a susceptible patient can be alleviated. In the six patients in which such prophylaxis was used the mean clinical score was 2.6. All six patients recovered without specific treatment, and in five patients there was no evidence of pneumonitis or renal impairment. The heart and lung transplant recipient in whom unequivocal evidence of primary CMV pneumonitis was obtained remained clinically well and made a complete recovery receiving hyperimmune globulin prophylaxis without the need for additional treatment. In similar patients elsewhere, in whom hyperimmune globulin prophylaxis was not used, primary CMV infection was more severe (mean clinical score 8.06 ), and there was a significant mortality $(20 \%){ }^{7}$ Interestingly, the two patients in our study who received a CMV positive graft without hyperimmune globulin prophylaxis had more severe disease (clinical scores of 8 and 7).

Our results in this small, non-randomised, study suggest that primary $\mathrm{CMV}$ infection can not be prevented by hyperimmune globulin prophylaxis, but it can be modified. All six patients given prophylaxis developed CMV specific IgM within three months, denoting a primary infection. These findings are similar to those obtained in a recent prospective trial of hyperimmune globulin prophylaxis after renal transplantation. ${ }^{8}$ Randomised studies of the protective effect of $\mathrm{CMV}$ specific hyperimmune globulin should now be undertaken in patients who receive heart, lung, and heart and lung transplantation to confirm our results. The incidence and severity of primary CMV infection has led others to advocate avoiding the implantation of a heart from a positive donor into a susceptible host. ${ }^{9}$ With this strategy, organs from CMV antibody positive donors are "matched" to CMV antibody positive recipients and potential recipients without CMV antibody must await the offer of an organ from a similarly seronegative donor. Recent evidence that patients known to possess CMV IgG antibody before transplantation can undergo clinically important "reinfection" when engrafted with an organ from a CMV antibody positive donor ${ }^{9}$ is cause for a reappraisal of the "avoidance strategy". The incidence of serious illness in the patients in whom CMV reinfection occurred as a result of donor transmission was less than that which typically occurs in donor transmitted primary CMV infection, but it is clear that the matching of organs from CMV antibody positive donors to CMV antibody positive recipients does not eliminate clinically important illness due to donor transmitted CMV infection.

The currently inadequate donor pool inevitably means that rigid adherence to an "avoidance strategy" will deny some cardiac transplant patients the possibility of transplantation. While our modest experience requires validation by others, our results suggest that a less cautious outlook is justified. Prophylaxis with hyperimmune globulin offers a method of using CMV positive organs in susceptible patients if clinical need demands it.

Our alternative strategy relies on the performance of a sensitive and specific test for CMV specific antibodies in both donor and recipient serum on the day of operation. The results of these tests allow prophylactic CMV specific hyperimmune globulin in CMV antibody negative recipients to be used rationally and cost effectively. This is particularly important because hyperimmune globulin is expensive (currently around $£ 3,000$ per patient course at commercial prices). The modest cost of performing ELISA tests for CMV IgG, even out of hours, will be more than offset by mitigating the effects of the disease and by restricting prophylatic globulin to those seronegative patients in whom it is absolutely necessary.

Our studies have been limited to CMV 
antibody negative recipients and have no direct bearing on the problem of reinfection following the engraftment of an organ from a CMV antibody positive donor into a CMV antibody positive recipient. It has been shown, however, that such infections are due to the donor virus strain, ${ }^{9}$ and this implies that the recipients' preformed antibody is not protective against the donor strain. If it proves possible to characterise donor and recipient strains before engraftment-for instance, by "fingerprinting" the CMV DNA present in the lymphocytes of donor and recipient-such results might form the basis of selective use of prophylactic hyperimmune globulin in CMV antibody positive patients receiving organs from CMV antibody positive donors. The breadth of antibody specificity in CMV specific hyperimmune globulin, manufactured from a very large donor pool, may provide protection against antigenically diverse strains. The alternative, of giving prophylactic hyperimmune globulin to all such patients, would be extremely expensive.

We are greatly indebted to the Northern Regional Blood Transfusion Service (Messrs A Reid and R Masterman) for the supply of CMV negative blood and blood products. We also thank Dr Hugh Lloyd, consultant haematologist, for his considerable help in obtaining supplies of CMV specific hyperimmune globulin.

1 Glenn J. Cytomegalovirus infections followng renal transplantation. Rev Infect Dis 1981;3:1151-78.

2 MacKinnon S, Burnet AK, Crawford RJ, Cameron S, Leask EGS, Sommerville RG. Seronegative blood products prevent primary cytomegalovirus infection after bone marrow transplantation. J Clin Pathol 1988;41:948-50.

3 Stirk PR, Griffiths PD. The use of monoclonal antibodies for the diagnosis of cytomegalovirus infection by the detection of early antigen fluorescent foci (DEAFF) in cell culture. J Med Virol 1987;21:329-37.

4 Ganciclovir: British National Formulary. London: British Medical Association and the Royal Pharmaceutical Society, 1989;233-4

5 Schafers H-J, Haverich A, Wahlers T, Milbradt H, Flik J, Fieguth HG, Borst HG. Cytomegalovirus prophylaxis after heart transplantation using specific hyperimmunoglobulin. Transplant Proc 1987;19:4061-2.

6 Smiley ML, Wlodaver CG, Grossman RA, et al. The role of pretransplant immunity in protection from cytomegalovirus disease following renal transplantation. Transplantation 1985;40:157-61.

7 Wreghitt TG, Hakim M, Gray JJ, Kucia S, Wallwork J, English TAH. Cytomegalovirus infection in heart and heart and lung transplant recipients. J Clin Pathol 1988; 41:660-7.

8 Snydman DR, Werner BG, Heinze-Lacey B, et al. Use of cytomegalovirus immune globulin to prevent cytomegalovirus disease in renal transplant recipients. $N$ Engl $J$ Med 1987;317:1049-54.

9 Grundy J, Super M, Sweny P, et al. Symptomatic cytomegalovirus infection in seropositive kidney recipients: reinfection with donor virus rather than reactivation of recipient virus. Lancet 1988;ii:132-5. 